\title{
Anomalous Low-Frequency Noise Increase at the Onset of Oscillations in Gunn Diodes
}

\author{
Ó. García-Pérez, I. Íñiguez-de-la-Torre, S. Pérez, \\ J. Mateos, T. González \\ Departamento de Física Aplicada \\ Universidad de Salamanca \\ Salamanca, Spain \\ ogarcia@usal.es
}

\author{
Y. Alimi, A. Song \\ School of Electrical and Electronic Engineering \\ University of Manchester \\ Manchester, United Kingdom
}

\begin{abstract}
In this work, the presence of anomalous lowfrequency noise effects in Gunn diodes has been studied and evidenced. Near the onset of Gunn instability, there is a limited range of bias voltages for which the noise significantly increases at frequencies well below the frequency of oscillation. For higher bias voltages, the oscillation becomes purer and such low-frequency noise drastically vanishes.
\end{abstract}

Keywords- Gunn devices; III-V semiconductor materials; microwave oscillators; noise measurement.

\section{INTRODUCTION}

Gunn diodes have been commonly used in the implementation of microwave oscillators. Nowadays, due to the advances in fabrication techniques and semiconductor materials, Gunn devices working up to several hundreds of $\mathrm{GHz}$ can be found in the literature [1]-[3], and even some recent studies predict structures and topologies prospectively able to oscillate at $\mathrm{THz}$ frequencies [4].

A Gunn diode oscillates when a bias voltage above certain threshold is applied to the device. However, between the initial static state and the generation of a purely periodic signal there exists a voltage range for which incoherent fluctuations appear. In the last case, when uncorrelated instabilities are formed, one of the effects that can be observed is an anomalous increase of the noise at very low frequencies. Moreover, this anomalous low-frequency noise disappears at higher bias when the oscillation peak is well established. This phenomenon was reported decades ago in some experimental works based on GaAs devices [5]-[6], although restricted to measurements in a very limited frequency range. More recently, the study of the low-frequency noise preceding the Gunn oscillations is found potentially useful for the characterization of mm-wave and $\mathrm{THz}$ Gunn sources. In this line, the effect has also been reproduced using Monte Carlo particle simulations for a variety of devices working above $100 \mathrm{GHz}$, including InN, InGaAs, InP and GaN diodes [7]-[9].

The origin of such critical enhancement of the lowfrequency noise is attributed to the existence of intermittent oscillations for a given range of voltages, preceding the formation of a coherent oscillation. In other words, the coexistence of both oscillatory and non-oscillatory states occurring with a random period leads to an anomalous increase of the low-frequency noise at the onset of the pure oscillation [6],[10]. The objective of this work is to analyze this phenomenon in more detail by combining both Monte Carlo simulations and experimental measurements performed with InGaAs diodes.

\section{Monte Carlo Simulations}

In a first approach, the noise characteristics of a Gunn diode have been studied by means of a home-made software tool based on the Monte Carlo particle method. The simulated device is a one-dimensional InGaAs diode, with an active region of $4 \mu \mathrm{m}$ length and doped $2 \times 10^{16} \mathrm{~cm}^{-3}$. A notch has been introduced at $1 \mu \mathrm{m}$ distance from the cathode to facilitate the formation of the oscillatory domains.

The simulated current spectral densities for different DC voltages are shown in Fig. 1. The fundamental frequency of oscillation is around $50 \mathrm{GHz}$. When no bias is applied to the device, the spectrum is flat and corresponds to thermal noise. For a bias voltage $V=1.6 \mathrm{~V}$, the power begins to concentrate around the oscillation frequency. At the same time, the flat noise density at lower frequencies also increases due to thermal effects. When a voltage of $1.65 \mathrm{~V}$ is applied, the oscillation peak appears much clearer, whereas a prominent noise hump arises at low frequencies. Further increasing of the bias voltages makes the oscillation peak sharper. However, the low-frequency noise hump counterintuitively disappears.

The phenomenon of interest can be clearly appreciated in Fig. 2. In the graph, the current noise density obtained at 3 $\mathrm{GHz}$ (low-frequency) for different bias voltages has been plotted. In a narrow range between $1.6 \mathrm{~V}$ and $1.65 \mathrm{~V}$, when the high-frequency instabilities appear, the low-frequency noise rapidly grows up. Above this range, the oscillation is well established and the low-frequency noise abruptly vanishes. Despite its simplicity, these simulation results are very illustrative since they allow us to determine that the previous effect is not due to non-idealities in the physical device or issues related with an experimental setup, but inherent to the mechanisms present during the origination of the Gunn fluctuations. 


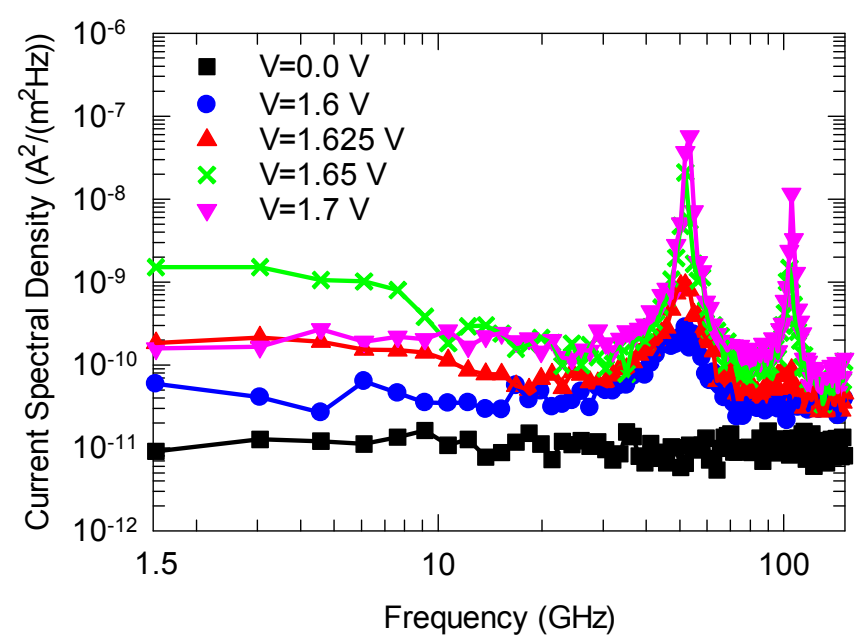

Fig. 1. Simulated current spectral density of a 1D InGaAs Gunn device for different DC voltages.

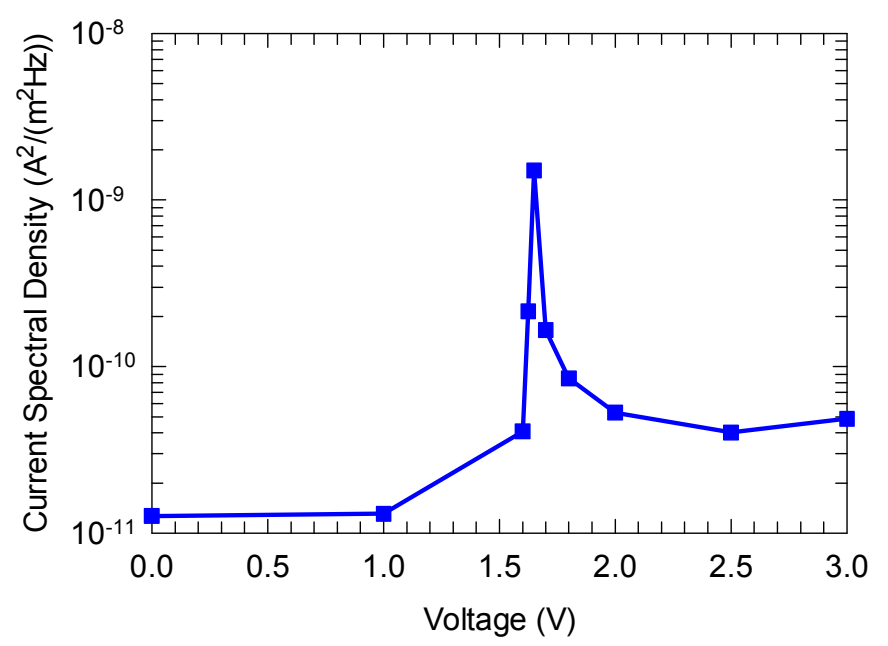

Fig. 2. Simulated noise current spectral density of the $1 \mathrm{D}$ InGaAs Gunn device at $3 \mathrm{GHz}$.

\section{EXPERIMENTAL MEASUREMENTS}

In order to experimentally validate the previous results, the power spectra of a set of Gunn diodes have been measured using a vector network analyzer with noise measurement capabilities, model PNA-X N5244A with Option 029 from Agilent Technologies, in the frequency range between 10 $\mathrm{MHz}$ and $43.5 \mathrm{GHz}$. This equipment allows acquiring the output power density from an electronic component in a wide frequency range and with high levels of accuracy and sensitivity [11]. The devices are InGaAs planar diodes, based on a structure similar to the one presented in [1]. A scheme of the measurement setup is depicted in Fig. 3. The reference plane is placed at the end of the access coplanar transmission line after a proper calibration step. It means that the losses due to the probe and the cable are not taken into account in the results.
The measured power spectra of a diode with $3.1 \mu \mathrm{m}$ channel length are shown in Fig. 4. The frequency of oscillation, which is proportional to the length of the device, corresponds to $30 \mathrm{GHz}$ in this case. When there is no current in the device the output is flat. As it is expected, the power density is approximately $-174 \mathrm{dBm} / \mathrm{Hz}$, which corresponds with the thermal noise of a passive device at $300 \mathrm{~K}$ [12]. Up to $V=5 \mathrm{~V}$, due to the current flowing through the device, both flat and $1 / f$ noise progressively increases. At $V=5.5 \mathrm{~V}$ some instabilities begin to appear in the upper part of the graph. Close to $V=8 \mathrm{~V}$, when the oscillation peak begins to be formed, a noticeable noise hump appears in the low part of the spectrum at around $400 \mathrm{MHz}$. It is remarked that such noise does not correspond to the well-known thermal or Flicker types, since its behavior and frequency dependence is of a completely different nature. Above this threshold, for $V=8.7$ $\mathrm{V}$, the maximum power of the oscillation increases, but the low-frequency noise hump disappears.

The noise power density as a function of the voltage is represented in Fig. 5 for the frequency of $400 \mathrm{MHz}$, where the anomalous noise hump presents the maximum. The response is in essence equivalent to the one obtained from simulations in Fig. 2. In a first range, below $V=6 \mathrm{~V}$, the diode does not oscillate and the low-frequency noise is of $1 / f$ nature, showing a response proportional to the bias current. In the subsequent region, that can be established up to $V=8.5 \mathrm{~V}$, the device begin to present some high-frequency instabilities. In such regime, the anomalous low-frequency noise hump grows up to almost two orders of magnitude over the $1 / f$ noise level, due to the presence of incoherent high-frequency fluctuations. In the last region, above $V=8.5 \mathrm{~V}$ the oscillation is purer and the lowfrequency noise level significantly decays.

Finally, it must be noted that an equivalent response has been found in more samples of similar length. As far as the device oscillated, a noise hump with more or less magnitude appeared for certain range of voltages. In other cases, probably due to manufacturing defects, the devices did not oscillate but only thermal and $1 / f$ noise effects were observed.

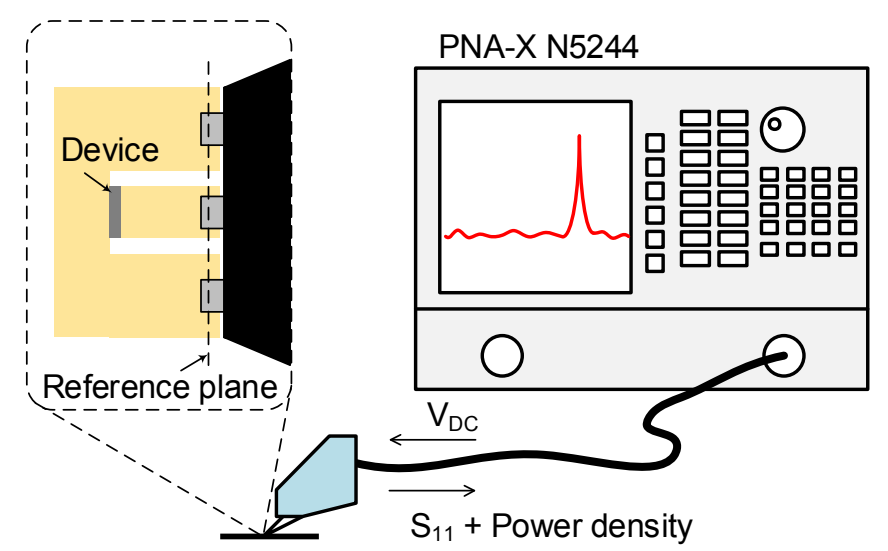

Fig. 3. Measured noise power spectral density of the $3.1 \mu \mathrm{m}$ length planar Gunn diode at $400 \mathrm{MHz}$. 


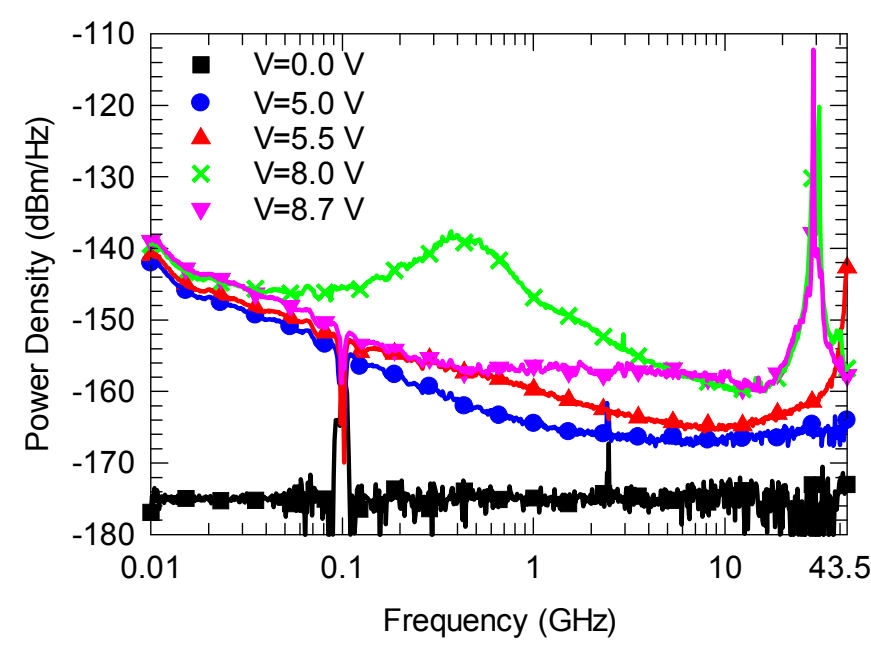

Fig. 4. Measured power spectral density of a $3.1 \mu \mathrm{m}$ length planar Gunn diode for different DC voltages.

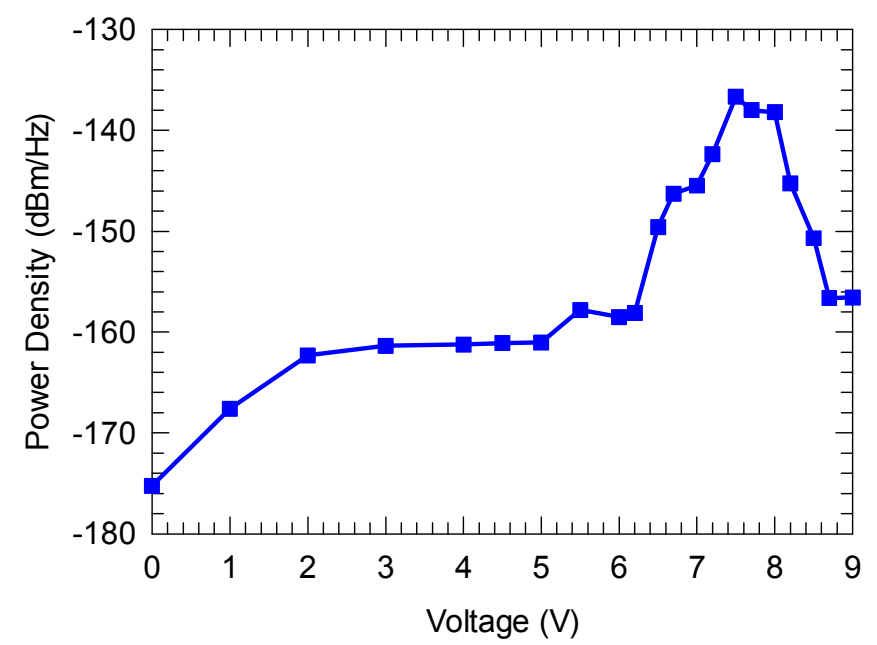

Fig. 5. Measured noise power spectral density of the $3.1 \mu \mathrm{m}$ length planar Gunn diode at $400 \mathrm{MHz}$.

\section{CONCLUSIONS}

The presence of a type of low-frequency noise during the initiation of oscillations in Gunn devices has been thoroughly analyzed. Both simulated and experimental results exhibit a similar behavior and have been useful to further understand the noise mechanisms that are present in Gunn diodes at the onset of oscillations. In practice, this knowledge can be used as an indirect method to predict higher-frequency oscillations in setups where a direct detection is a complicated challenge, as in the case of (sub-) THz Gunn oscillators

\section{Acknowledgment}

This work has been partially supported by the Spanish MINECO through project TEC2013-41640-R, and by the Consejería de la JCyL through project SA052U13. Ó. GarcíaPérez wants to thank the European Social Fund (ESF) for financing his postdoctoral contract.

\section{References}

[1] C. Li, A. Khalid, S. H. Paluchowski-Caldwell, M. C. Holland, G. M. Dunn, I. G. Thayne and D. R. S. Cumming, "Design, fabrication and characterization of $\mathrm{In}_{0.23} \mathrm{Ga}_{0.77} \mathrm{As}$-channel planar Gunn diodes for millimetre wave applications," Solid-State Electron., vol. 64, pp. 67-72, 2011.

[2] A. Khalid, C. Li, V. Papageorgiou, G. M. Dunn, M. J. Steer, I. G. Thayne, M. Kuball, C. H. Oxley, M. Montes Bajo, A. Stephen, J. Glover and D. R. S. Cumming, "I $\mathrm{I}_{0.53} \mathrm{Ga}_{0.47}$ As Planar Gunn Diodes Operating at a Fundamental Frequency of $164 \mathrm{GHz}$, , IEEE Electron Device Lett., vol. 34, pp. 39-41, Nov 2013.

[3] A. Khalid, G. M. Dunn, R. F. Macpherson, S. Thoms, D. Macintyre, C. Li, M. J. Steer, V. Papageorgiou, I. G. Thayne, M. Kuball, C. H. Oxley, M. Montes Bajo, A. Stephen, J. Glover and D. R. S. Cumming, "Terahertz oscillations in an $\mathrm{In}_{0.53} \mathrm{Ga}_{0.47} \mathrm{As}$ submicron planar Gunn diode," J. Appl. Phys., vol 115, p. 114502, 2014.

[4] S. Pérez, T. González, D. Pardo, J. Mateos, "Tehahertz Gunn-like oscillations in InGaAs/InAlAs planar diodes," J. Appl. Phys., vol. 103, p. 094516, 2008.

[5] K. Matsuno, "Critical fluctuation in GaAs in DC electric field," Phys. Lett. A, vol. 31, pp. 335-336, Mar. 1970.

[6] S. Kabashima, Y. Yamakazi, T. Kawakubo, "Critical fluctuation near threshold of Gunn instability,” J. Phys. Soc. Jpn., vol. 40, pp. 921-924, Apr. 1970.

[7] E. Starikov, P. Shiktorov, V. Gruzinskis, L. Reggiani, L. Varani, J. C. Vaissiere, "Noise Enhancement as Indicator of Instability Onset in Semiconductor Structures," AIP. Conf. Proc., vol. 780, pp. 791-794, 2005.

[8] P. Shiktorov, E. Starikov, V. Gruzinskis, L. Varani, L. Reggiani, "Giant enhancement of low-frequency noise as precursor for the onset of a high-frequency instability," AIP Conf. Proc., vol. 1129, pp. 179-182, 2009.

[9] A. Íñiguez-de-la-Torre, I. Íñiguez-de-la-Torre, J. Mateos, T. González, "Correlation between low-frequency current-noise enhancement and high-frequency oscillations in GaN-based planar nanodiodes: A Monte Carlo study," Appl. Phys. Lett., vol. 99, p. 062109, 2011.

[10] Ó. García-Pérez, Y. Alimi. A. Song, I. Íñiguez-de-la Torre, S. Pérez, J. Mateos, T. González, "Experimental assessment of anomalous lowfrequency noise increase at the onset of Gunn oscillations in InGaAs planar diodes," App. Phys. Lett., vol. 105, p. 113502, 2014.

[11] Agilent Technologies Inc., "High-accuracy noise figure measurements using the PNA-X series network analyzer," Application Note 1408-20 (5990-5800EN), 2013.

[12] D. M. Pozar, Microwave Engineering, NY: John Wiley and Sons, 1998, p. 551 . 